\title{
The Development and Utilization of Flash Software
}

\author{
Wang Yan $^{1, a}$ \\ ${ }^{1}$ Animation Institute, Hebei Institute of Fine Art, ShiJiaZhuang, 050700, China \\ Yanwang006@163.com
}

Keywords: Flash ; Animation Design; ActioScript

\begin{abstract}
With the development of The Times, we has entered the era of intelligence, the development and utilization of FLASH software, not only in making the simple animation, but also has developed in constant innovation with of the era of intelligence .Getting understand of writing FLASH animation ActioScript not only greatly enhance the interactivity of FLASH animation, users can control the play and stop on its own animations, at the same time with ActioScript statements can create design animation effects. Making us more in-depth understanding of development and utilization of flash animation.
\end{abstract}

\section{Introduction}

One: What is the FLASH. FLASH has three layers meaning: (1) The English meaning of FLASH for photography is an instant, transient.(2) It is relatively popular in the computer animation software of design and manufacture; (3) It represents the popular creation made with the software in the Internet, television. Flash is a kind of interactive multimedia technology, his predecessor is Futureplash, early popular online plug-in for making vector animation. Now there have been hundreds of thousands of online Flash site, the famous special ShockRave sites which belongs to Macromedia, all adopt the Shockwave Flash and Director. We can say that Flash has grown to become known quantity standard about interactive. It's the mainstream in the future on web page. Flash is launched the excellent web animation design software by MACROMEDIA company in June 1999 in the United States. It is a kind of tool for interactive animation design, it can innovatively interfaces music, sound, animation to be together, in order to produce high quality dynamic web pages effect.

Two: Why use FLASH software. FLASH software is one of the necessary learning animation professional software, used to draw the picture, make the action, and can also write code at the background, this is a the software that can be applied to paintings guys can also be applied to a software programmer, adapt widely, and easy to show the effect, convenient and quick, so powerful is obvious to FLASH software, more and more people join to use this software, FLASH software combined with popular completely, because it is easy to use, so the popular elements can be placed into the FLASH production in the era of intelligence.

The characteristics of the FLASH:

(1)The use of vector graphics and streaming technology. What's the Vector graphics different to bitmap graphics is that it don't change image quality along with the zoom in and out of video, so the flash-makings can be played on any resolution, this is one of it's a big advantage; Streaming technology allows the animation to play while downloading, thus relieve the waiting mood for web browsers.(2)Easy to download. The generated animation (. SWF) file in software production is very small, a few $\mathrm{K}$ bytes of animation files has been achieved amazing animation effects. Using that on the web design can not only make the web more vivid, and small and exquisite for downloading quickly. Making animation can be opened in a short period of time and be able to play.(3)The various elements interact together, is another important feature of FLASH software, more and more people have the Flash As the preferred tool when for animation design, and produced many breathtaking animation (film) effect. For example: the early pleasant goat and grey Wolf, that's children's favorite, so Flash software has become a fashion icon of the new method in intelligent era.(4)The powerful animation design in Flash, realizing interactivity through the ACTION and FS COMMAND, making design process has more degrees of freedom, in addition, it has cooperate very well with today's most popular Dreamweaver web design tools, can be directly 
embedded in web pages, and at any place.

\section{The composition structure of the interface and basic principle}

One: interface. Flash interface composited by 4 parts, the menu bar, the main toolbar ,scenario and stage.(1)the main toolbar: using the tools in the main toolbar can draw animation effects what we want.(2)The timeline: the timeline used to organize and control the file content in a certain period of time, the timeline can be divided into the control layer and a time line control area, the major components about timeline is the main component layer, frame and playback head.layer control area: located in the left side of the timeline. Layer just like slide film stacked together, each layer contains a show of different images on the stage.The timeline control area: located in the right side of the timeline, is made up of frame, the play needle and multiple button as well as an information bar. Like film, Flash document divides time length into frames. Frame contained in each layer displayed on the right side of the time line. At the top of the timeline indicates a frame number title. The play needle indicates the stage in the current frame.(3)Scenario and stage: the scene is the largest activity space, like a full length drama, all animation include more than one scenario.The scene is what we often say the stage, is a rectangular area where we editing and playing animation .(4)And a menu bar is where all the control button in the FLASH software can be found in it.

Two: The basic principle. The animation made by frame and tweening. FLASH use the principle of animation., cut each picture into the frame. The animation the generated when broadcast quickly .but due to the high compression of the flash document, the file is very small. It also can be programmed to control the animation playing, which is easier to control the animation. This is the basic principle of flash animation.

\section{Production process of flash animation}

One: The animation script : We need a complete animation script before we do the animation。

Two: Character design: We can design the model using drawing tool in flash on the scenario and stage before pondering the meaning expressed by the animation script. Three: Scene design: also be made in Flash software. The scene design is the critical step in shaping the film character and style. We should draw scene pictures before reading and understanding the script, mastering the historical background, knowing the characteristics of the Times, deeping in life, collecting materials, keeping the style of scene and character unified. Four: Drawing script: The scenario and stage also can be drawn directly dynamic script in FLASH. The function of script is changing the text to be story. The script is one of the most important part of the whole animation. What we should pay attention is when making animation, we should use good camera language, telling story interesting, lens joining naturally. Five: Drawing element: Drawing elements in Flash scenarios and stage, and then put the components in to library for using them at any time. Six: The original painting: the element such as scenario and model, produced them into original painting. Seven : Export images: we export PNG images frame by frame, the purpose is to add special effects on the frames. Eight: Add special effects: Flash software usually combined with AE, which can make a good animation, making a stronger visual perception.

\section{Knowing ActioScript of Flash in intelligent era}

ActioScript: ActioScript is the language used on the flash scripting, more used on the developed interactive, entertaining, practical. We can also add logic and other control to the documentation of the media elements use the ActioScript code, in order to write out the interactive applications suitable for user's. This is a big advantage of the flash.

One:actionscript terms: (1) Action:is the instructions which playing file give according to the action editing code. (2)The event: The action when files in play. For example, in loading movie clip, head into the frame, the user clicks a button Or movie clip, or the user press the keys on the 
keyboard, can produce different events. (3) Class: class can be created to define new types of data types. If you want to define the class, need to use the class keyword in the external script files (4) Constructor: the constructor is used to define the class attributes and methods of function.(5)The event handler: It's a special action manage such as mouse down event or the load events. There have two classes for a total of Actionscript event handler: the event handler method and event listener. In the toolbox "action", each actionscript object wether with event handler method or event listener has been named "Events" or "Listeners" subcategory. Some commands can be used for the event handler as well as be used for event listener, and included in the above two sub-categories.(6) Example: Instance belongs to a class object. Each instance include all the attributes and methods of this class (7) Instance name: instance name is used to the only way to represent in the film clips and button .You can use the "properties" panel to specify name for the instance on the stage. (8) Methods: the method is a function associated with the class.(9) Object: The object is a collection of properties and methods, and each object has its own name.(10)Package: The package is located in the specified class path,it contains one or more class files directory. (11) Property: Property is to define the characteristics of the object. (12) Target path: the target path is hierarchical structure address about the object include instance name in SWF files, variables.

Two: ActioScrip writing. (1)When writing scripts, there have two ways, one is writing directly in "action" panel, code script written here directly stored in the FLA file. Where we are going to give instructions, where to write code, there are two ways to write code.1) Click the frame directly, Which frame we want to give command,we click the frame and then press F9, then enter input the code.2) Or directly click on the element, and then press F9 enter the code.(2) The instance for animation script writing

For example: the role of the "video demo" button is to load the "video demo" sub-page, button action script is as follows:

On (release) \{

Load Movie (" video demo. SWF, "_root);

\}

Video play button: the sub-pages of "video demo" provide video clips such as people walking, running, jumping, click on the corresponding button then the player loading the video files which convert to FLV format in advance. Such as the role of "people walk " button is to demonstrate the "people walk .FLV" video, button action script is as follows:

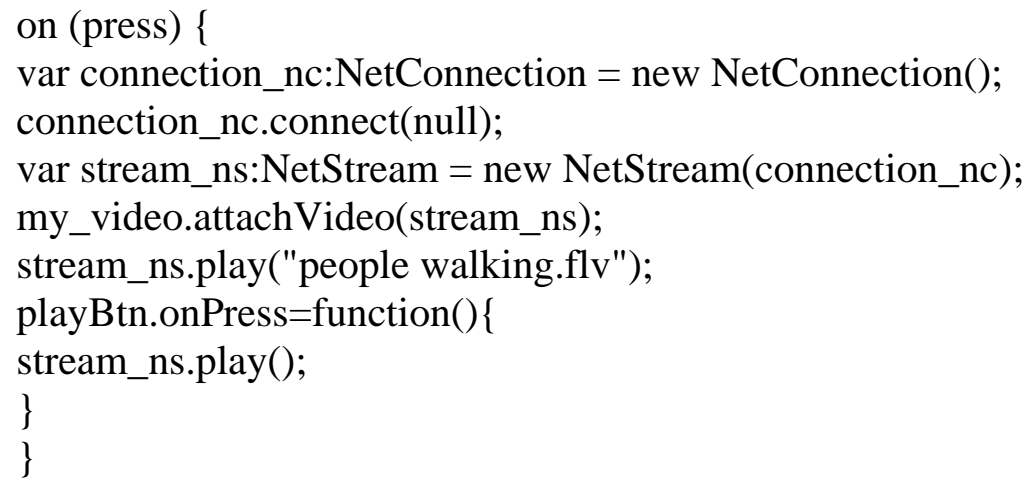

Three: The characteristics when write animation script:(1)Should to use the function, statement or other language elements, can be directly double click on the corresponding project in the movement to the toolbox, or click add new project to the script above the script pane button, then select the corresponding project.(2) Syntax highlighting.(3)Reminder display code.(4)Syntax checking .(5)Determine the task object.

\section{Debugging scripts}

FLASH can find errors when play the SWF files use the "debugger" provided by the system.

Select "control | debugging menu" to open the debugger, the debugger shows hierarchical display list about the movie clips in the current load to the Flash Player, use a debugger, can modify 
the value of the variable and attribute play the video, and you can use breakpoints to stop SWF files and line by line to track the action script code. In addition, users can also use the output panel display an error message and variable and object list, if you want to observe the change of a variable, should add the trace statements in the script first, and then select "control $\mid$ debugging film" menu, entering in the status of film test, the system will automatically open the output panel, and displays a variable value and the changes.

\section{Summary}

Along with the development of the Flash software, its function are constantly extend and expand in the era of intelligence, at the same time, we can use the Flash animation effect reach a diversifying works. Flash is not only create animation on painting and script code, it can be requested on a higher level of demand. It can be used cooperate with other software to get the effect we want. In any case, I would like to clear that as long as able to take both AS programming skills and outstanding creativity into account, it can create a lot of amazing work. In other words, only to achieve a good combination about animation design drawing and programming language, to create our own perfect animation language.

\section{References}

[1] WuYiXian, WuMuCheng . Brilliant Flash MX Chinese programming. Beijing .Publishing House Of Electronics Industry.2003

[2] YangDongYun. Solution for Common Problems In Flash courseware manufacture technology . Science and the Mass,2010(5) :112-113.

[3] ChenHuiAn. JavaScript Web Page Creation Research[M].Beijing. Posts and Telecom Press.2002 